\title{
Dynamic axial crushing of short to long circular aluminium tubes with agglomerate cork filler
}

\author{
C.P. Gameiro*, J. Cirne \\ CEMUC - Departamento de Engenharia Mecânica, Faculdade de Ciências e Tecnologia da Universidade de Coimbra, 3030-788 Coimbra, Portugal
}

Received 20 September 2006; received in revised form 18 January 2007; accepted 26 January 2007

Available online 6 February 2007

\begin{abstract}
Cork is a complex natural cellular material with quite unknown or not well understood properties. It is available in the natural and in the agglomerate form and it is an ecological and very durable material. That is why it is used today as thermal and acoustic insulator, as a seal and as an energy-absorbing medium in flooring, shoes and packaging, among others. However, the application of agglomerate cork as filler inside structural thin-walled sections, in order to increase the energy absorption, has not been much explored. Dynamic experimental tests were carried out on empty and micro-agglomerate cork-filled tubes with 22 and $50 \mathrm{~mm}$ in internal diameter $(D)$ and length $(L)$, respectively, and numerical simulations were performed with the finite element method software LS-DYNA ${ }^{\mathrm{TM}}$, showing good agreement in terms of load-displacement curves and deformation patterns. Having validated the numerical model with experiments, the finite element model was used to undertake a systematic study of circular tubular structures impacted at $10 \mathrm{~m} / \mathrm{s}$. The load-deformation characteristics, energy-absorption response and collapse mode transitions of empty and cork-filled aluminium tubes with varying diameters and thicknesses $(t)$, lengths of 25,300 and $350 \mathrm{~mm}$, but with constant slenderness ratios $D / t$ and $D / L$ were thus studied. Relevant comparisons were raised, showing that the slenderness ratios are very important parameters that globally govern the percentage increase in energy absorbed by tubular structures after cork-filling during an impact loading.
\end{abstract}

(C) 2007 Elsevier Ltd. All rights reserved.

Keywords: Impact; Cork; Aluminium tubes; Energy absorption; Finite element method; Mechanical behaviour

\section{Introduction}

Energy-absorbing systems of various kinds are being extensively used in many engineering applications such as automobiles and other transport vehicles. Indeed, during an impact event, the energy of the crash and the manner in which the loads are transmitted through the system, are very important points. As a consequence, the axial crushing behaviour of thin-walled structures, which are efficient energy absorbers, has been a topic of great interest for many researchers. Experimental, theoretical and numerical studies on axial structural collapse have mainly focused on the mode of collapse, the peak force, the mean force and the energy-absorption characteristics.

Steel has been extensively used in energy-absorbing systems, due to low prices combined with excellent

\footnotetext{
${ }^{*}$ Corresponding author. Tel.: + 351239790762 ; fax: + 351239790701.

E-mail address: celina.gameiro@dem.uc.pt (C.P. Gameiro).
}

ductility. But the importance of the reduction of structural components mass has increased in the recent years. Hence, the use of aluminium tubes with different shapes and geometries, and thus energy-absorbing efficiencies, has been frequently promoted [1,2]. Furthermore, computational mechanics has been an important tool in the assessment of the crash behaviour of various structures since numerical simulations enable new design and materials to be evaluated without extensive testing. For example, Langseth et al. [3] validated the LS-DYNA ${ }^{\mathrm{TM}}$ computer code using an experimental database obtained by axial crushing of square thin-walled aluminium tubes in alloy AA6060, and performed a parametric study of the response of square aluminium tubes, varying the mass of the projectile and the impact velocity.

Many authors examined the parameters which could influence the crushing pattern of square and cylindrical tubes under quasi-static and dynamic axial loading since collapse mode transitions of thin tubes strongly influence 
the energy absorption. Abramowicz and Jones [4] explored the critical parameters which govern the transition from global bending to progressive collapse, for square and circular thin-walled mild-steel columns subjected to quasistatic and dynamic axial loading. Besides, the stress wave propagation phenomena during an axial impact was also focused in the work published by Karagiozova and Jones [5-7]. Recently, Gupta et al. [8] showed that transitions in the collapse mode of circular aluminium tubes may be caused by thickness eccentricity around the tube circumference, or by the nonaxisymmetry in the tube end conditions.

The question of whether the performance of axially crushed tubes could be improved by introducing a filler material has also been asked several times during the past two decades. Santosa et al. [9] found that the increase of the mean crushing force of a filled column has a linear dependence with the foam compressive resistance and cross-sectional area of the column. The mechanical behaviour of different types of foams used as filler in aluminium tubular structures, under static and dynamic loading, has been studied extensively in the last few years $[10,11]$. Generally, there is a significant increase in the average crushing force and, in some cases, a change of the deformation behaviour is observed because of the interaction effect between the tube and the foam [12]. Santosa et al. [13] also demonstrated that filling a square aluminium box column may be preferable to thickening the column wall. Aktay et al. [14], among others, developed a numerical study of the crushing of thin-walled aluminium tubes with foam used as filler and validated adequate models for filled structures.

Cork is a quite complex natural cellular material with quite unknown or not well understood properties. The fundamental aspects of the mechanical behaviour of cork under static compressive loading have already been discussed in several studies [15]. However, the mechanical behaviour of natural and agglomerate cork when subjected to axial impact has been investigated very recently, when quasi-static (at $10^{-3} \mathrm{~s}^{-1}$ ) and dynamic (strain rates from 200 to $600 \mathrm{~s}^{-1}$ ) uniaxial compressive tests performed on natural and agglomerate cork were compared by the authors $[16,17]$.

Hence, the purpose of this study is to analyse the energyabsorption behaviour of thin aluminium tubes filled with agglomerate cork subjected to uniaxial impact loading. Thus, numerical simulations were carried out using the finite element code LS-DYNA ${ }^{\mathrm{TM}}$, based on a model that globally showed good agreement with the experimental results. The validated numerical model was used to study the load-deformation characteristics, energy-absorption response and collapse mode transitions of empty and cork-filled aluminium tubes with varying diameters and thicknesses, lengths of 25,300 and $350 \mathrm{~mm}$, but with constant slenderness ratios $D / t$ and $D / L$, impacted at $10 \mathrm{~m} / \mathrm{s}$. The aim of this systematic study was to relate and better understand the dynamic behaviour of short to long aluminium tubes filled with agglomerate cork, in order to consider possible innovative mechanical applications in energy-absorption fields, combining those two materials.

\section{Materials and structures}

\subsection{Materials}

The structures studied in this paper were circular aluminium tubes of different geometries, empty and filled with micro-agglomerate cork. The aluminium alloy considered (more than $99 \% \mathrm{Al}$ ) is in accordance with the standard AW 6060/6063 and was submitted to a T5 heat treatment. It has been characterised by means of uniaxial tensile tests performed at a crosshead speed of $1 \mathrm{~mm} / \mathrm{min}$ in a hydraulic testing machine INSTRON 4204 (Fig. 1). The alloy tested presented a $0.2 \%$ proof stress of $150 \mathrm{MPa}$ approximately.

Natural cork, as found in Quercus Suber cork oaks, is extremely difficult to use in industrial applications because of its short size and its micro-structural heterogeneity, which generates very variable mechanical properties. As a consequence, the authors used micro-agglomerate cork containing $0.5-2 \mathrm{~mm}$ cork particles, mixed with polyurethane adhesive, latex, paraffinic oil and paraffin.

Micro-agglomerate cork shows a characteristic compressive stress-strain curve, similar to that of closed-cell cellular solids, composed of three distinct regions: elastic, collapse and densification. At low strains, the cork deforms elastically. The collapse region is characterised by a plateau stress increasing with strain. Finally, at a critical strain, the cells start to touch each other and the stress increases sharply. Micro-agglomerate cork has some characteristics of an efficient energy absorber since it presents a long stress-strain curve up to densification at relatively high strains. Fig. 2 shows the compressive uniaxial stress-strain curve for micro-agglomerate cork at quasi-static and dynamic strain rates. Further details on the experimental technique and data analysis carried out to obtain the curves are part of the published work by Gameiro et al. [17]. The study mentioned showed that the stress values increased 2-3 times those of quasi-static strain rate test when the

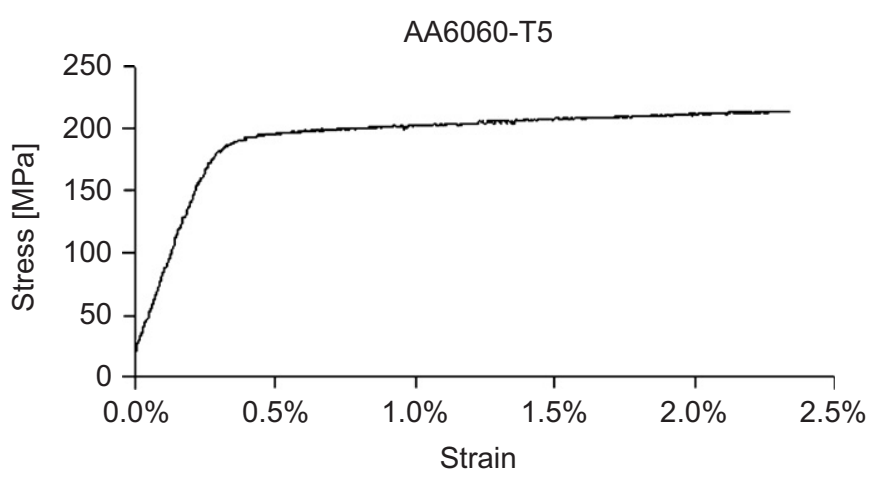

Fig. 1. True stress-strain curve of the aluminium alloy AA6060. 


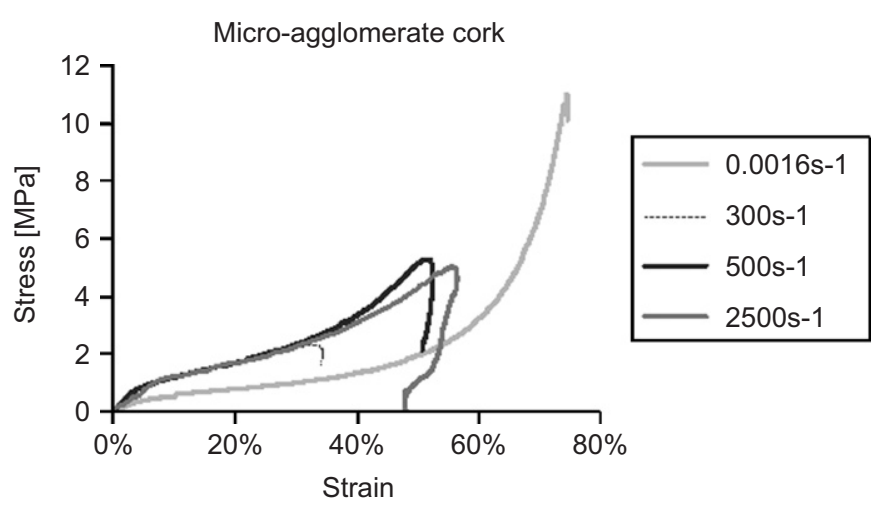

Fig. 2. Compressive uniaxial stress-strain curves for micro-agglomerate cork at quasi-static and dynamic strain rates.

strain rate increased to the high strain rate deformation range. The increase was supposed to be mainly due to the different features unique to the dynamic crushing of cellular solids that can drive the cork strength upwards: localisation, micro-inertia and shock enhancement caused by densification at high strain rates. Within the dynamic range, from 200 to $2500 \mathrm{~s}^{-1}$, the micro-agglomerate cork did not show substantial differences in the compressive curves obtained. As also reported in Ref. [17], there is an increase of the Young's modulus when quasi-static and dynamic curves are compared.

\subsection{Structures}

The tubular structures simulated numerically had a circular section. Indeed, axisymmetric and circular shapes seem to provide the widest range of all choices for use as absorbing elements because of their favourable plastic behaviour under axial forces, as well as their common occurrence as structural elements. In fact, circular tubes under axial compression are reported to be the most prevalent components in energy-absorbing systems since they provide a reasonably constant operating force. Furthermore, circular tubes have comparatively high energy-absorbing capacities and feature a favourable stroke length per unit mass. In comparing lateral compression vs. axial compression, the axial buckling mode has a specific energy-absorbing capacity that is approximately 10 times greater than that of the same tube when it is compressed laterally between flat plates. This is due to the fact that, during axial loading all wall material in a tube can be made to participate in the absorption of energy by plastic work [18].

One of the main objectives of this paper is to describe and quantify the increase in energy absorption consequent from the introduction of cork filler inside circular tubes with varying geometric parameters. It is known that when thin-walled metal tubes are filled with a lightweight foam core, an interaction effect usually exists between the tube wall and the foam filler. Mainly due to this effect, the crushing loads of foam-filled tubes are higher than the sum of the crushing loads of foam alone and tube alone. At the same time, a shift in the collapse mode from diamond into concertina with foam-filling was observed, for example, in Al foam-filled $\mathrm{Al}$ and steel tubes, polyurethane and polystyrene foam-filled Al tubes [1,2,9-14,22]. Besides, it is known from experimental tests that the slenderness ratios $D / t$ (internal diameter to thickness ratio) and $D / L$ (internal diameter to length ratio) are the primary parameters controlling the buckling pattern of tubes $[19,20]$. Expecting that the contribution of the filler in a cork-filled tube by means of interaction effect also varies with the deformation mode characteristic of the tube when empty, emphasis was put on the influence of those same slenderness ratios on the energy-absorption capacities of tubular structures filled with cork, impacted at $10 \mathrm{~m} / \mathrm{s}$ by a rigid wall.

The mass of the rigid wall was chosen for each tube so that the energy of the impact provided a deformation of the tubes of at least $75 \%$ (Table 1). In a first part, the tubes simulated had lengths of 25,300 and $350 \mathrm{~mm}$ and constant slenderness ratios $D / t$ and $D / L$ of, respectively, 25, 38 and 50 , and $0.19,0.25$ and 0.88 . In a second part, and in order to investigate if the tendencies observed were still valid for tubes with a length randomly fixed, $D / L$ equal to 0.60 , and $D / t$ equal to 25,38 and 50 , additional circular tubes were simulated (Table 1). Consequently, a total of 24 structures were studied.

The tubes were catalogued through the attribution of specific names in the form $(D / t) C(100 \times D / L) F$, where $C$ stands for "circular", $F$ for "filled", and $D, t, L$ are, respectively, the internal diameter, thickness and length of the tube considered. Hence, $25 \mathrm{C} 19$ is a circular tube with $D / t$ equal to $25, D / L$ equal to 0.19 and unfilled. The same tube filled with micro-agglomerate is called 25C19F.

Table 1

Geometry of the tubes simulated

\begin{tabular}{lllrlll}
\hline Tube & $D(\mathrm{~mm})$ & $t(\mathrm{~mm})$ & $L(\mathrm{~mm})$ & $D / t$ & $D / L$ & Striking mass $(\mathrm{kg})$ \\
\hline 25C19(F) & 66.5 & 2.66 & 350 & 25 & 0.19 & 2000 \\
25C25(F) & 76 & 3.04 & 300 & 25 & 0.25 & 1500 \\
25C88(F) & 22 & 0.88 & 25 & 25 & 0.88 & 40 \\
38C19(F) & 66.5 & 1.75 & 350 & 38 & 0.19 & 1500 \\
38C25(F) & 76 & 2 & 300 & 38 & 0.25 & 500 \\
38C88(F) & 22 & 0.58 & 25 & 38 & 0.88 & 20 \\
50C19(F) & 66.5 & 1.33 & 350 & 50 & 0.19 & 1500 \\
50C25(F) & 76 & 1.52 & 300 & 50 & 0.25 & 500 \\
50C88(F) & 22 & 0.44 & 25 & 50 & 0.88 & 20 \\
25C60(F) & 50 & 2 & 83.3 & 25 & 0.6 & 150 \\
38C60(F) & 80 & 2.1 & 133.3 & 38 & 0.6 & 500 \\
50C60(F) & 60 & 1.2 & 100 & 50 & 0.6 & 500 \\
2-38C19(F) & 76 & 2 & 400 & 38 & 0.19 & 1500 \\
2-38C88(F) & 44 & 1.16 & 50 & 38 & 0.88 & 100 \\
\hline
\end{tabular}




\section{Finite element modelling and validation}

The geometrical models used in this analysis were created with the pre-processor ETA Femb and then exported to LS-DYNA ${ }^{\mathrm{TM}}$, version 970 , to carry out numerical solutions. This code uses an explicit algorithm based on central difference method, which is particularly adequate to the simulation of impact events. The visualisation tool processors used were ETA PostGL and ETA Graph.

\subsection{Properties of the materials}

The success of the numerical finite element codes for analyses of structures and components incorporating cork rests upon the accuracy and efficiency of the applied material model. Unfortunately, a given material model is rarely capable of capturing accurately the mechanical behaviour of the material for all existing load configurations. Moreover, the commercially available material models in LS-DYNA ${ }^{\mathrm{TM}}$ are generally black boxes, only recognised by the required input and a limited model description, with no information on how the models are programmed and implemented. Based on these facts, it is imperative to set priorities and strategies for the choice of the material model applied to a material subjected to a given load configuration, knowing that none of the models available perfectly fits. The strategy was to use a simple and generic model, able to produce a stable and efficient numerical solution for large strains and displacements. Moreover, as the dynamic uniaxial stress-strain behaviour of micro-agglomerate cork was assessed in recent investigations, it was also important to choose a model:

- allowing the input of the dynamic uniaxial compressive stress-strain curve;

- with known required inputs;

- and able to simulate the uniaxial confined and unconfined compression of micro-agglomerate cork with very good results.

Since the recovery of cork after unloading is not relevant for the processing of the energy-absorption values during the dynamic compression of the micro-agglomerate cork, model MAT_HONEYCOMB seemed the most adequate to the problem raised even if it implied permanent strains. It allows introducing the experimental curve, supposes a zero Poisson's ratio and captures perfectly the behaviour of a typical cellular solid confined in a tube and impacted uniaxially, as referenced in the published work by Hanssen et al. [21]. Other material models exist in the code for foams or foam-like and rubber-like materials, but they are inadequate to model cork since they tend to be dedicated to specific applications or often suppose that the material is incompressible. The *MAT_HONEYCOMB is adequate to honeycomb and foam materials with real anisotropic behaviour. The behaviour before compaction is orthotro- pic where the components of the stress tensor are uncoupled, i.e., a component of strain will generate resistance in the local 1-direction with no coupling to the local 2 and 3 directions. This behaviour is in agreement with the assumption that the Poisson coefficient of the material is nearly zero. The elastic moduli vary from their initial values to the fully compacted values, linearly with the relative volume (defined as the ratio of the current volume to the initial volume). The parameters introduced, some of them found in the literature [15], were the density $\rho\left(293 \mathrm{~kg} / \mathrm{m}^{3}\right)$, the Young modulus $E(9 \mathrm{GPa})$ and Poisson coefficient $v(0.3)$ of the compacted material, as well as the stress-strain curve of the cellular material for dynamic loadings [17].

*MAT_PIECEWISE_LINEAR_PLASTICITY was the material model used for the aluminium tubes. This material models an elastic-plastic material with an arbitrary stress-strain curve and an arbitrary strain rate dependency can be defined.

The data from the tensile curve previously defined were used. Strain rate was accounted for, using the Cowper-Symonds model which scales the yield stress of the material with the factor $\left[1+(\dot{\varepsilon} / C)^{1 / P}\right]$, where $C$ and $P$ are constants depending on the material. For aluminium alloys, the values adopted were $P=4$ and $C=6500 \mathrm{~s}^{-1}$, since the strain sensitivity of aluminium is small [23].

\subsection{Numerical modelling}

For the micro-agglomerate cork, the meshing consisted of 8-node hexahedral solid elements with 3 degrees of freedom per node, for eight points of integration resulting in full integration, with no hourglass problems. A topology mesh was performed with Eta pre-processor, in order to mesh cork with $1 \mathrm{~mm}$ elements for the shorter tubes and $3 \mathrm{~mm}$ elements for the longer, along the volume. The circular aluminium tubes under compressive loading were drawn by their middle surface in $3 \mathrm{D}$ in order to capture the nonsymmetric deformation patterns, despite the increase of CPU time. In this case, 1 or $3 \mathrm{~mm}$ Belytshko-Tsay shell elements were used, with seven integration points along the thickness. No gap was introduced between the cork and the tube.

The boundary conditions adopted for the tubes correspond to the introduction of two modelled rigid walls placed at the ends of the tubes. One of them is fixed, whereas the other impacts the material while associated to a mass and a velocity. The velocity introduced was $10 \mathrm{~m} / \mathrm{s}$, and the mass was chosen so that the final deformation of the tube was always at least $75 \%$ of the initial length.

For each tubular structure (with or without filler), the Automatic_Single_Surface contact of the LS-DYNA ${ }^{\mathrm{TM}}$ library was used to prevent self-penetration of the lobes during deformation and the Automatic_Surface_to Surface contact permitted to avoid the mutual penetration of the two coupled materials. No friction was considered between the tube and the cork since the contact with the 
micro-agglomerate cork considered contains paraffin, an effective lubricant. An internal solid anti-collapse contact was used additionally in order to prevent numerical problems that could arise when the solid elements of foam were heavily compressed and distorted. The option helps to reduce excessive compression; therefore, avoids too large drops in the stable solution time steps. This option also helps to avoid too large element distortions that may lead to ill-defined elements causing negative volume. For the tubes, the displacements of the nodes in contact with the compression rigid walls were not allowed, except the displacement of the upper nodes along the impact axis, in order to avoid nonrealistic deformation modes.

\subsection{Experimental validation}

The validation procedure applied to material models can usually be divided in three distinct steps: a level of calibration tests where the strategy is to use information from experimental tests for the initial calibration of the material models; a level of nonuniform tests with different loading conditions for validation of the global model behaviour; finally, a numerical validation in connection with structural interaction. In that case, it is intended to check if the material models used for the cork and the aluminium tube allow obtaining numerical results for the filled structures in agreement with the experimental ones, in the specific case of a uniaxial dynamic loading, in order to justify the execution of a systematic numerical study with longer filled tubes. Therefore, only the first and the third level of the usual material model validation procedure were carried out.

First, numerical analyses of the dynamic uniaxial compressive behaviour of the micro-agglomerate cork were conducted. Fig. 3 relates numerical simulations with the corresponding experimental data at $500 \mathrm{~s}^{-1}$. A good agreement is visible in the curves. Secondly, in order to calibrate the aluminium material model and perform the numerical validation corresponding to the structural interaction of both cork and aluminium materials, experimental dynamic tests were performed on empty and corkfilled short tubes in a drop-weight apparatus with a hammer mass of $25.308 \mathrm{~kg}$ equipped with a Kistler quartz transducer, type 9341B. Circular empty and cork-filled tubes with the characteristics displayed in Table 2 rested on a plate which in turn was fixed to a solid base. At least three empty and cork-filled samples were tested.

The experimental and numerical force-displacement curves of the empty and filled tubes are shown in Fig. 4. As seen, the agreement is quite good. The percentage increase in energy absorption due to the filling of the tubes, which is the main parameter studied in this paper, is very similar when obtained numerically and experimentally (Fig. 5). For example, the absorbed energy difference between the empty and cork-filled tubes at $60 \%$ strain (maximum strain reached experimentally) is $55.5 \%$ based

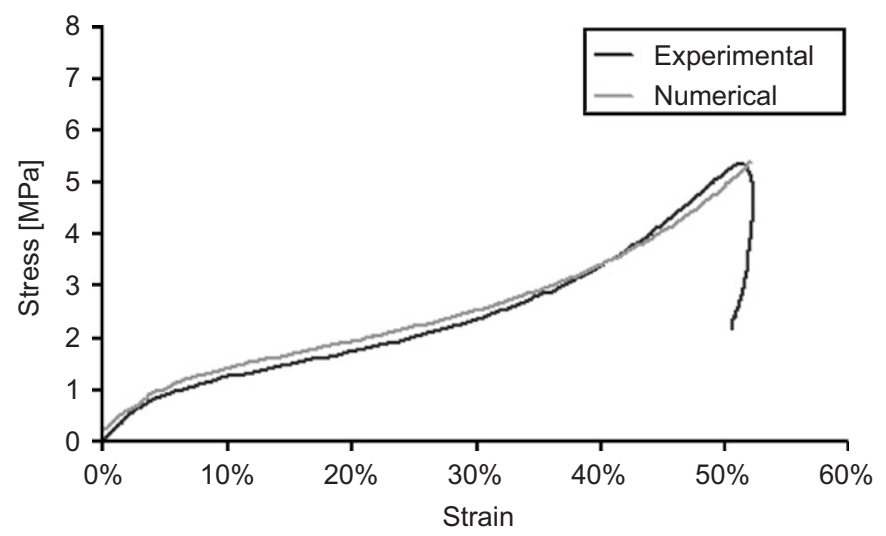

Fig. 3. Experimental and numerical stress-strain curves for microagglomerate cork at $500 \mathrm{~s}^{-1}$.

Table 2

Geometry of the tubes tested in the drop-weight apparatus

\begin{tabular}{lllllll}
\hline Tube & $D(\mathrm{~mm})$ & $t(\mathrm{~mm})$ & $L(\mathrm{~mm})$ & $D / t$ & $D / L$ & Impact velocity \\
\hline T50-0.5(F) & 22 & 0.5 & 50 & 44.0 & 0.44 & $3.9 \mathrm{~m} / \mathrm{s}$ \\
\hline
\end{tabular}

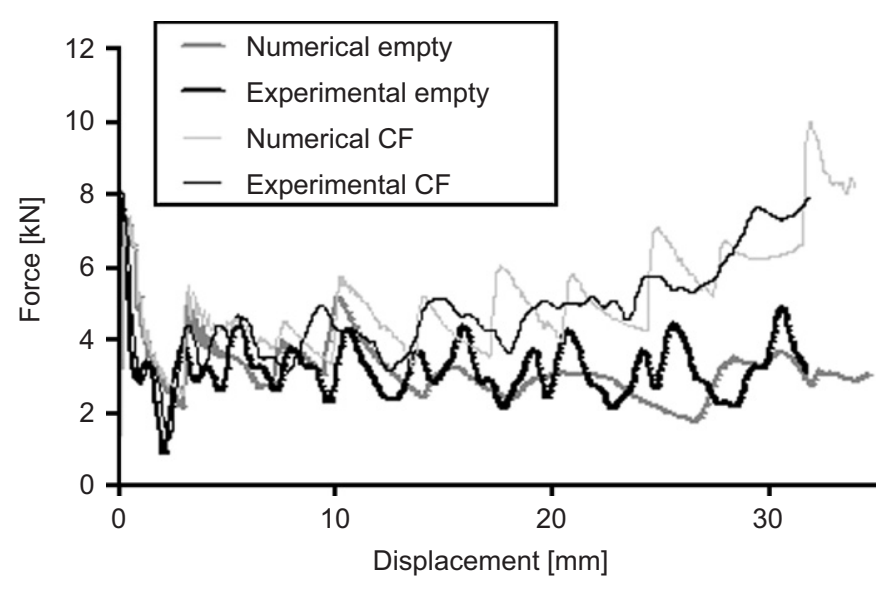

Fig. 4. Experimental and numerical force-displacement curves for the empty and cork-filled (CF) tubes tested in the drop-weight apparatus.

on experimental and numerical results. This fact suggests that the numerical model seems to capture quite correctly the contribution of the filler, the interaction effect and thus the increment of energy absorbed by the tubular structures at high strains after filling. Besides, the model deformed shapes (shift from diamond to concertina mode) and number of lobes also matched to the experimental deformed shapes, which again confirmed the agreement between model and experiments (Fig. 6). These conclusions allowed proceeding with confidence in the understanding of the geometrical parameters that may have a strong influence on the increment of energy absorbed by tubes after filling them with cork, based on the use of the material models and numerical modelling proposed. 


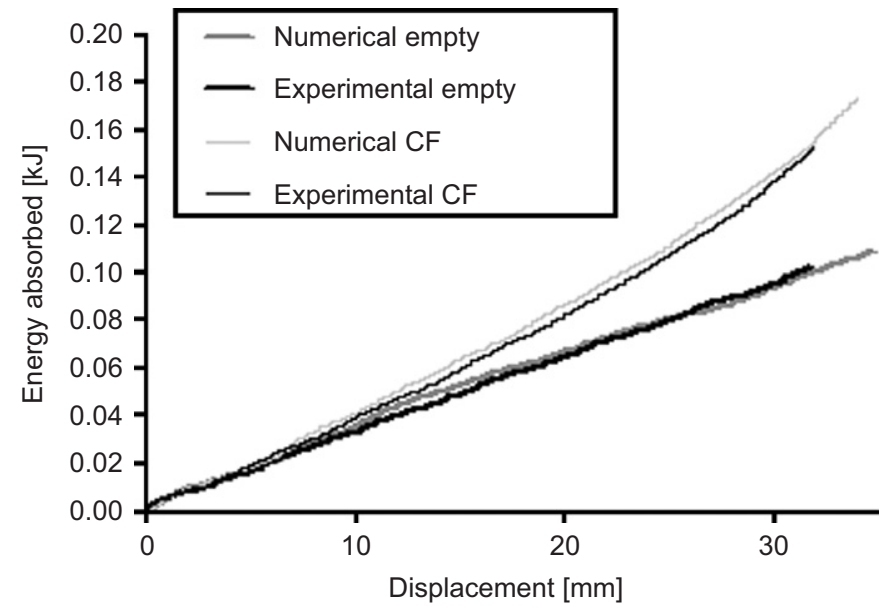

Fig. 5. Experimental and numerical energy-absorption curves for the empty and cork-filled (CF) tubes tested in the drop-weight apparatus.
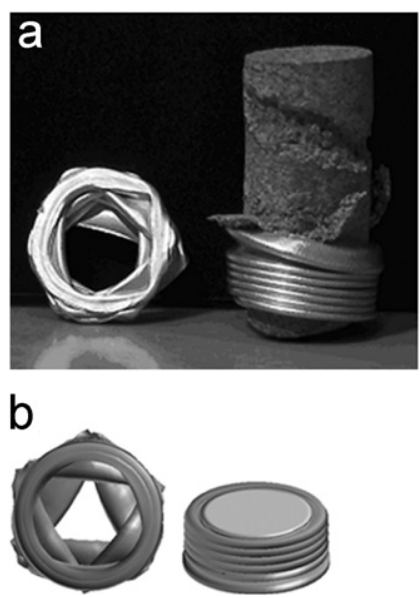

Fig. 6. Shape histories of the empty and cork-filled tubes tested in the drop-weight apparatus: (a) experimental and (b) numerical.

\section{Results and discussion}

\subsection{Example: results for $50 C 25$ and $50 C 25 F$}

The force-displacement, mean crushing load and absorbed energy curves were drawn for each tubular structure studied, up to $75 \%$ strain. The mechanical behaviour of the tubes is no longer relevant for higher strains, since the structure does not manage to keep its integrity. Figs. 7 and 8 show an example of force-displacement, average crushing load and energy- absorption curves calculated for the tubes $50 \mathrm{C} 25$ and $50 \mathrm{C} 25 \mathrm{~F}$. The absorbed energy $\left(E_{A}\right)$ and average crushing load $\left(P_{a}\right)$ of the tubes tested are closely related and were calculated using the following relation:

$P_{a}=\frac{E_{A}}{\delta}=\frac{\int P \mathrm{~d} \delta}{\delta}$,

where $P$ and $\delta$ are the load and displacement, respectively.

Fig. 7 shows the tubes wave-like oscillations of load. Cork-filling seems to considerably increase the force

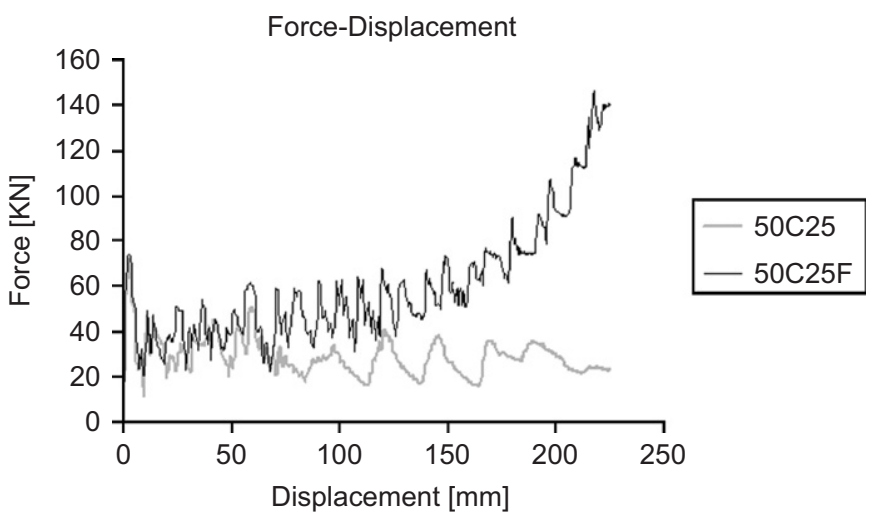

Fig. 7. Force-displacement curves for $50 \mathrm{C} 25$ and $50 \mathrm{C} 25 \mathrm{~F}$ tubes.

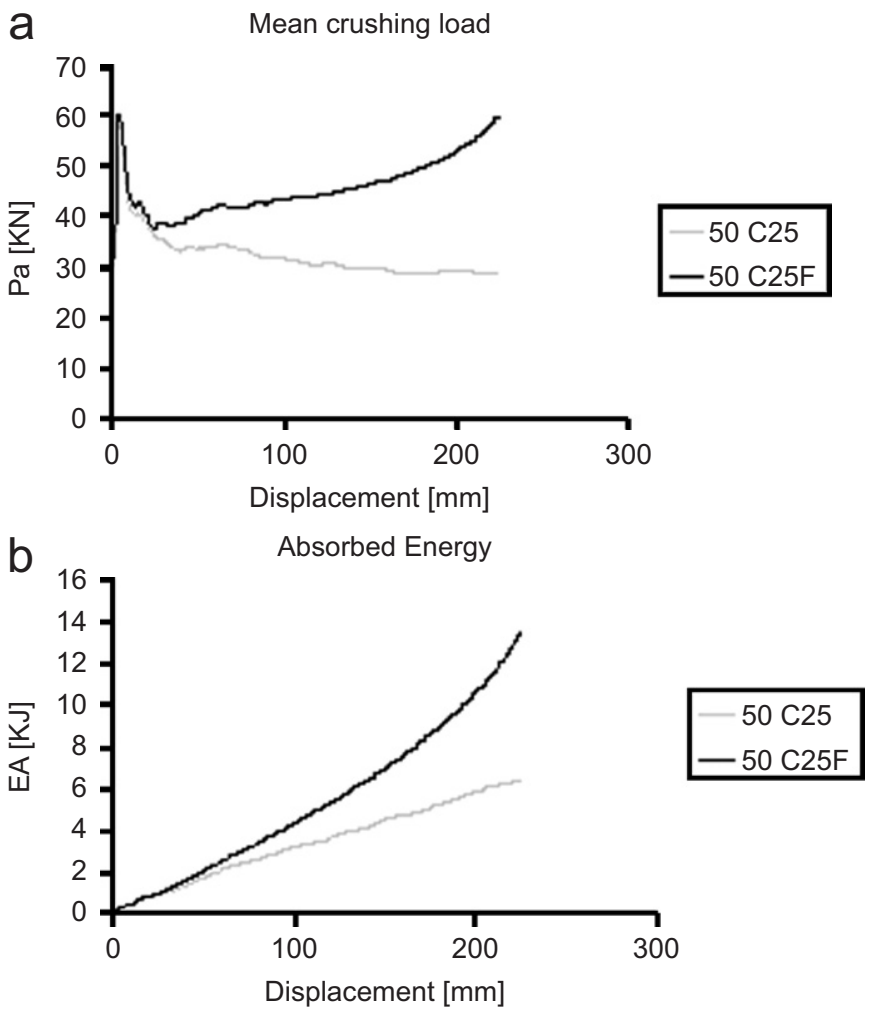

Fig. 8. Mean crushing load (a) and absorbed energy (b) for 50C25 and $50 \mathrm{C} 25 \mathrm{~F}$.

supported by the tube, especially for relatively high displacements, as also visible in Fig. 8(a). Whereas the empty tube mean crushing load is practically constant (after the first peak load) during all the deformation process, the same cork-filled tube presents a progressive increase of the load, possibly due to the interaction effect of both materials and to the densification of the filler at increasing deformation ratios. Indeed, many authors observed an increase in the force supported by foam-filled structures with increasing densities of the filler: higher the density of the filler, higher the resistance to tube folding [1,2,9-14,22,24]. At $75 \%$ strain, Fig. 8 shows filling increases the mean crushing load and the energy absorbed 
of approximately $110 \%$, which is very positive and encouraging for the emergence of innovative applications for micro-agglomerate cork, especially in crash applications. The larger the deformation of the structure, the larger the percentage increase in load and energy.

However, it is expected that the percentage increase in energy absorption due to cork-filling is dependent on the geometrical parameters of the tubes considered. In order to quantify the increase due to filling for each tube, emphasis is put on the percentage difference of energy absorbed (called PDEA) between the empty tube and the same corkfilled tube at $75 \%$ strain.

\subsection{PDEA vs. slenderness ratios}

For the tubes with lengths of 25,300 and $350 \mathrm{~mm}$ studied in a first part, the PDEA curves with varying strain (Figs. 9-11) as well as the PDEA values at $75 \%$ strain with varying slenderness ratios $D / t$ and $D / L$ (Fig. 12) were plotted for each tube.

Figs. 9-11 show that, for a fixed $D / t$, there is an increase in the PDEA with decreasing $D / L$ all along the deformation process. For the three values of $D / t$, the results are very similar when values of $D / L$ are proximal $(0.19$ and 0.25 ). Besides, for the same strain value and increasing $D / t$, there is also an increase in the PDEA value. In order to confirm this behaviour, more tubes were tested numerically with a ratio $D / L$ of $0.6 . D / t$ was once more fixed at 25,38 and 50 and $D, t$ and $L$ were chosen randomly so that the

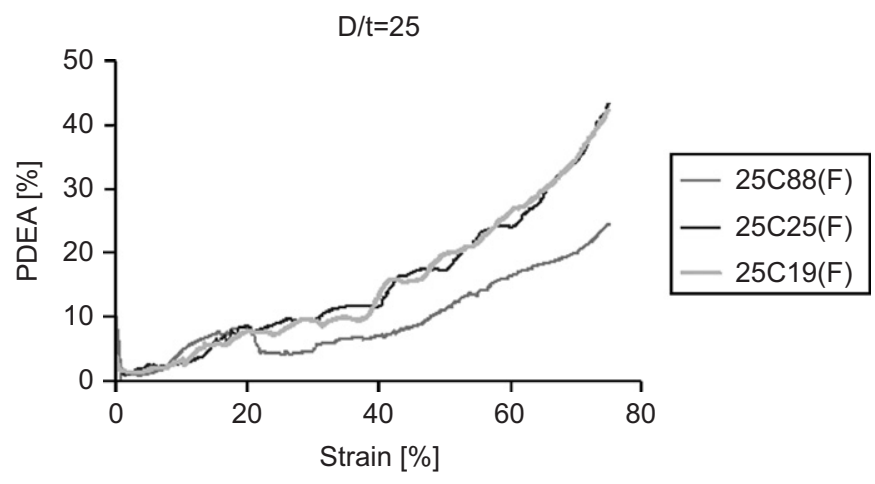

Fig. 9. PDEA for the tubes with $D / t=25$.

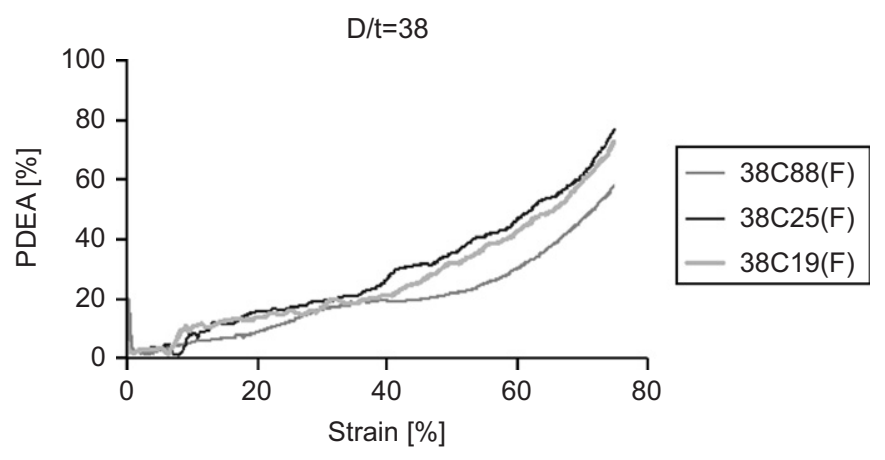

Fig. 10. PDEA for the tubes with $D / t=38$.

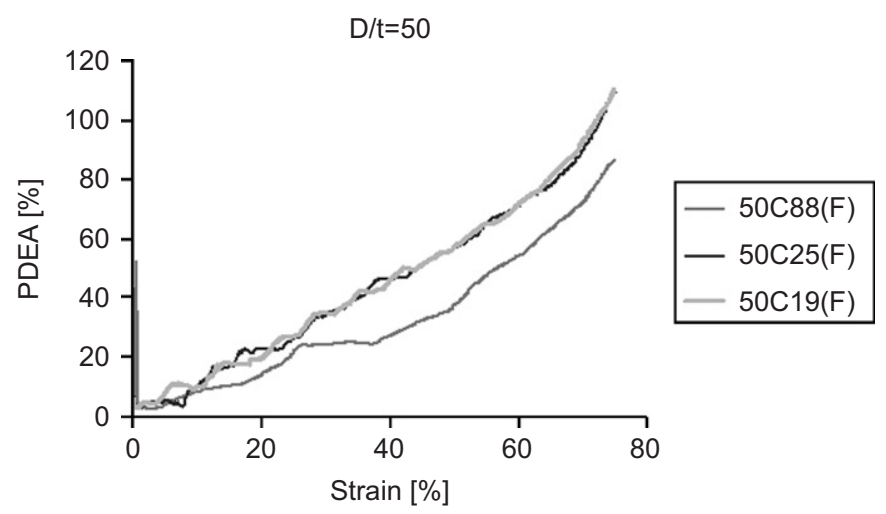

Fig. 11. PDEA for the tubes with $D / t=50$.

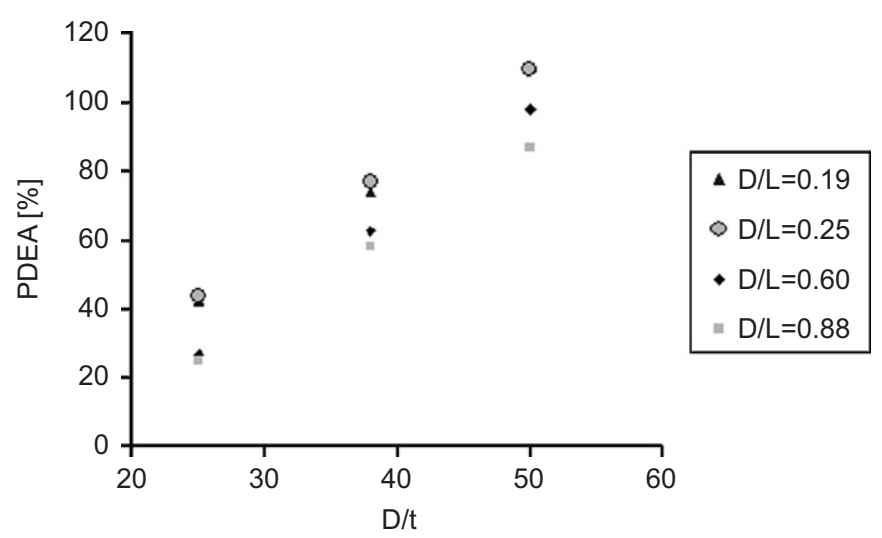

Fig. 12. PDEA values at $75 \%$ strain vs. $D / t$ and $D / L$.

previous conditions were observed and the tubes belonged to the same geometric range of the previous ones ( $L$ from 25 to $350 \mathrm{~mm}, D$ from 20 to $80 \mathrm{~mm}$ and $t$ from 0.4 to $4 \mathrm{~mm}$ ).

Fig. 12 summarises the variations of the PDEA at $75 \%$ strain with varying $D / t$ and $D / L$ for all the tubes simulated numerically. There is a pronounced increase in the PDEA with increasing $D / t$ and decreasing $D / L$ for all the tubes tested. Furthermore, for the range of tubes considered, the increase in PDEA values seems to be linear. Fig. 12 is in accordance with the fact that, for a fixed value of $D / L$, the PDEA increases with decreasing $t$. Moreover, it confirms that, for a fixed $D / t$, the PDEA increases with increasing $L$. The tubes simulated, in the range of geometric characteristics considered, absorb from $25 \%$ to $110 \%$ more energy after cork-filling, depending on the slenderness ratios and on the strain reached. As initially expected, the slenderness ratios are definitely parameters of extreme importance, controlling strongly the mechanisms of energy absorption subsequent to the introduction of the filler. The numerical results carried out in this paper are of great importance since they offer the possibility to predict the potential effects of filling in the energy absorbed, given a tube with known slenderness ratios, avoiding labour-intensive experimental tests. Numerical simulation happens to be a powerful tool in the quantification of the energy-absorption improvement 
provided by the introduction of cork inside circular aluminium tubes at $10 \mathrm{~m} / \mathrm{s}$.

\subsection{Interaction effect and deformation modes}

In a physical and mechanical point of view, there is an explanation for the increasing tendency observed in Fig. 12. Several mechanisms can be responsible for the increase in the crushing load after filling. One of them, mostly minor, is the crush resistance of the microagglomerate, which is summed to the crushing load of the empty tube. The most important contribution to the energy increase is predominantly due to an interaction effect between the tube wall and the micro-agglomerate cork. Mainly due to this effect, the crushing loads of the cork-filled tubes are higher than the sum of the crushing loads of the cork (alone) and the tube (alone). An illustration of this effect is shown in Fig. 13 for the tube $38 \mathrm{C} 25 \mathrm{~F}$. The filler contributes to provide a thickening and stabilising effect of the structure which directly increases the interaction effect and thus the energy absorption, as also referred in the work carried out by Reddy and Wall [22] on thin aluminium tubes filled with low density polyurethane foam. Besides, cork-filling reduces the fold length and hence increases the number of folds formed, which also contributes to an increase in the energy absorbed. With increasing $D / t$ and decreasing $D / L$, these effects are predominant, which explains why the energy absorption increases considerably after cork-filling in those cases.

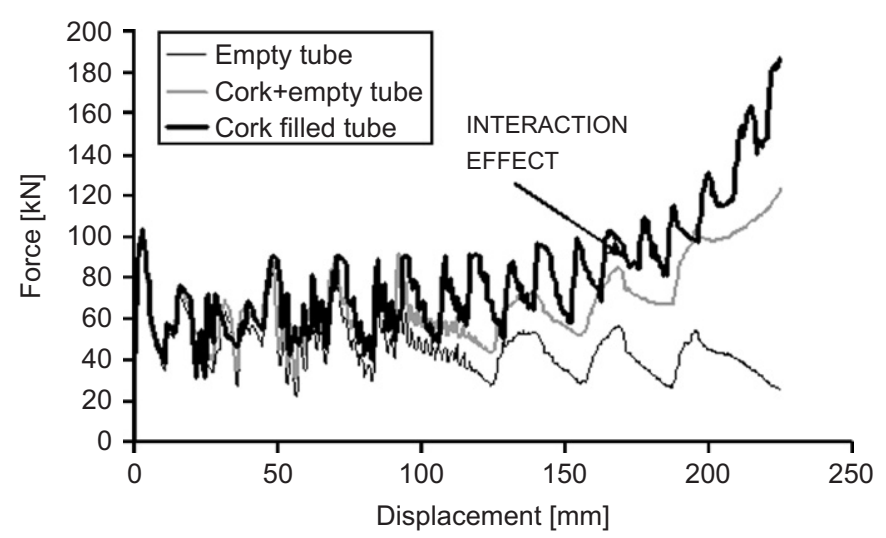

Fig. 13. Interaction effect observed for the tube $38 \mathrm{C} 25 \mathrm{~F}$.

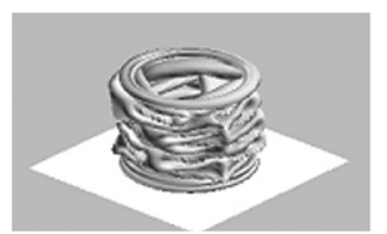

$50 C 19$

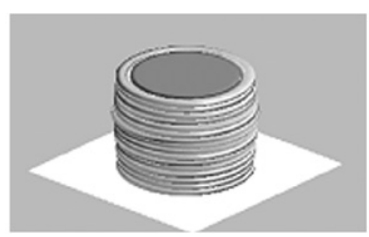

$50 \mathrm{C} 19 \mathrm{~F}$

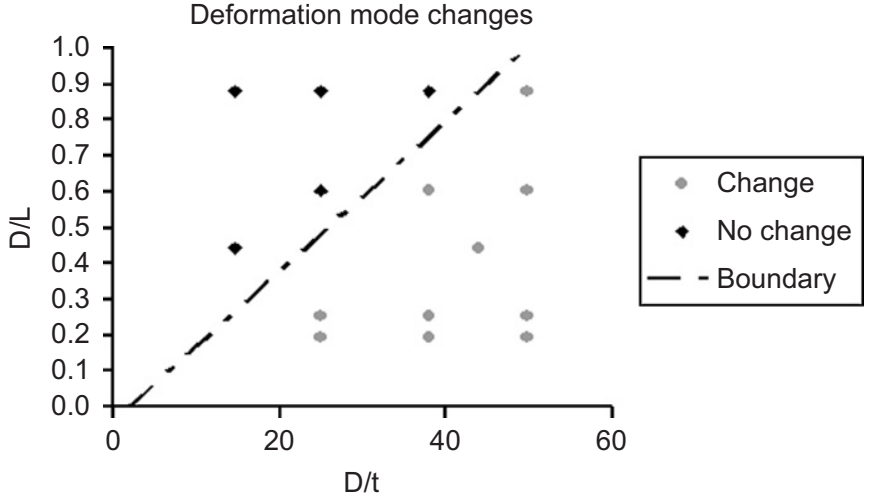

Fig. 15. Map of the deformation mode changes vs. the slenderness ratios of the tubes studied.

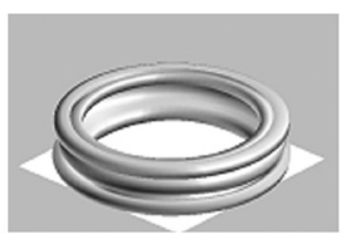

$38 \mathrm{C} 88$

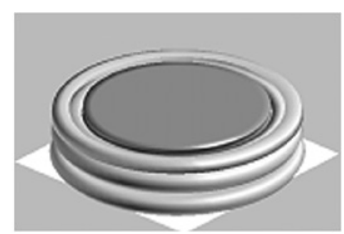

$38 \mathrm{C} 88 \mathrm{~F}$

Fig. 14. Examples of two tubes simulated with $(50 \mathrm{C} 19(\mathrm{~F}))$ and without $(38 \mathrm{C} 88(\mathrm{~F}))$ change of the deformation pattern after cork-filling. 


\section{Conclusions}

First of all, the numerical simulations performed with LS-DYNA ${ }^{\mathrm{TM}}$ suggested there are some tubular structures for which cork-filling may be very beneficial in terms of energy absorption, for impact loadings at $10 \mathrm{~m} / \mathrm{s}$. The increase of energy absorption was proved to be very dependent on the characteristic slenderness ratios of the tubes considered in the range studied.

As a consequence, this paper highlighted the fact that knowing the influence of those parameters on the PDEA is of extreme importance. The authors found that, in the range of $D, L$ and $t$ considered, there is an almost linear increase of the PDEA when $D / t$ increases, for a fixed $D / L$, and that the PDEA also increases with decreasing $D / L$, for a fixed $D / t$. In industrial applications, the possibility to predict the percentage increase of energy absorbed by aluminium tubes after cork-filling for impact loadings at $10 \mathrm{~m} / \mathrm{s}$ may be very important during the design of specific mechanical structures.

Those results also suggest that micro-agglomerate cork may become the adequate material for innovative applications that require low cost, low density and high energy absorption. Furthermore, micro-agglomerate cork presents similar mechanical behaviour when axially compressed, in a dynamic range from 200 to $2500 \mathrm{~s}^{-1}$, which suggests it may be a very interesting material to use in applications where strain rate sensitivity is undesirable.

More experimental and numerical tests must be performed, with larger ranges of geometric dimensions, in order to confirm if the tendencies observed remain valid. More adequate numerical models should also be programmed in order to better capture the viscoelasticity of cork and recovery of dimensions after impact.

\section{Acknowledgements}

The authors are grateful to the Portuguese Foundation for Science and Technology (FCT) who financially supported this work, through the scholarship SFRH/BD/ $18964 / 2004$, and to ROCAP for providing the cork samples.

\section{References}

[1] Hanssen AG, Langseth M, Hopperstad OS. Static and dynamic crushing of square aluminium extrusions with aluminium foam filler. International Journal of Impact Engineering 2000;24(4):347-83.

[2] Hanssen AG, Langseth M, Hopperstad OS. Static and dynamic crushing of circular aluminium extrusions with aluminium foam filler. International Journal of Impact Engineering 2000;24:475-507.

[3] Langseth M, Hopperstad OS, Berstad T. Crashworthiness of aluminium extrusions: validation of numerical simulation, effect of mass ratio and impact velocity. International Journal of Impact Engineering 1999;22(9-10):829-54.
[4] Abramowicz W, Jones N. Transition from initial global bending to progressive buckling of tubes loaded statically and dynamically. International Journal of Impact Engineering 1997;19:415-37.

[5] Jones N. Several phenomena in structural impact and structural crashworthiness. European Journal of Mechanics A-Solid 2003; 22(5):693-707.

[6] Karagiozova D, Jones N. Dynamic buckling of elastic-plastic square tubes under axial impact-II: structural response. International Journal of Impact Engineering 2004;30(2):167-92.

[7] Karagiozova D. Dynamic buckling of elastic-plastic square tubes under axial impact-II: stress wave propagation phenomenon. International Journal of Impact Engineering 2004;30:143-66.

[8] Gupta NK, Nagesh. Collapse mode transitions of thin tubes with wall thickness, end condition and shape eccentricity. International Journal of Mechanical Sciences 2006;48(2):210-23.

[9] Santosa SP, Wierzbicki T, Hanssen AG, Langseth M. Experimental and numerical studies of foam-filled sections. International Journal of Impact Engineering 2000;24(5):509-34.

[10] Hall IW, Ebil O, Guden M, Yu CJ. Quasi-static and dynamic crushing of empty and foam-filled tubes. Journal of Material Sciences 2001;36(24):5853-60.

[11] Kavi H, Toksoy AK, Guden M. Static and dynamic crushing of circular aluminium extrusions with aluminium foam filler. Materials and Design 2006;27(4):263-9.

[12] Toksoy AK, Guden M. The strengthening effect of polystyrene foam filling in aluminium thin-walled cylindrical tubes. Thin-Walled Structures 2005;43(2):333-50.

[13] Santosa S, Wierzbicki T. Crash behavior of box columns filled with aluminium honeycomb or foam. Computers and Structures 1998; 68(4):343-67.

[14] Aktay L, Toksoy AK, Guden M. Quasi-static axial crushing of extruded polystyrene foam-filled thin-walled aluminum tubes: experimental and numerical analysis. Materials and Design 2006;27(7): $556-65$.

[15] Fortes MA, Rosa ME, Pereira H. In: Cortiça A. IST Press; 2004.

[16] Gameiro CP, Cirne J, Gary G, Miranda V, Pinho-da-Cruz J, Teixeira-Dias F. Numerical and experimental study of the dynamic behaviour of cork. In: Teixeira-Dias F, Dodd B, Lach E, Schulz P, editors. Design and use of light-weight materials, third light-weight armour group workshop. Aveiro, Portugal; 2005. p. 65-84.

[17] Gameiro CP, Cirne J, Gary G. Experimental study of the quasi-static and dynamic behaviour of cork under compressive loading. Journal of Materials Science, 2007, in press, doi:10.1007/s10853-006-0675-6.

[18] Aljawi AAN. Numerical simulation of axial crushing of circular tubes. Journal of King Abdul Aziz University: Engineering Sciences 2002;14(2):3-17.

[19] Yamazaki K, Han J. Maximization of the crushing energy absorption of tubes. Structural Optimization 1998;16(1):37-46.

[20] Jensen O, Langseth M, Hopperstad OS. Experimental investigations on the behaviour of short to long square aluminium tubes subjected to axial loading. International Journal of Impact Engineering 2004;30(8-9):973-1003.

[21] Hanssen AG, Hopperstad OS, Langseth M, Ilstad H. Validation of constitutive models applicable to aluminium foams. International Journal of Mechanical Sciences 2002;44:359-406.

[22] Reddy TY, Wall RJ. Axial compression of foam-filled thin-walled circular tubes. International Journal of Impact Engineering 1988;7(2):151-66.

[23] Langseth M, Lademo OG. Tensile and torsion testing of AA6060-T4 and T6 aluminium alloys at various strain rates. Technical Report, Department of Structural Engineering, Norwegian Institute of Technology; 1994

[24] Toksoy AK, Guden M. The strengthening effect of polystyrene foam filling in aluminum thin-walled cylindrical tubes. Thin-Walled Structures 2005;43(2):333-50. 